\title{
The Nasal Oxygenation and Ventilation of the Airway (NOVA) Technique, a New and Safer Approach to Airway Management in the Critically Ill Patient
}

\author{
Steven Cataldo*, Michael Pedro and Banu Lokhandwala \\ Department of Anesthesia, SUNY Downstate Medical Center, 450 Clarkson Avenue, Brooklyn, NY 11203, USA
}

Received: October 04, 2014; Accepted: November 17, 2014, Published: December 05, 2014

*Corresponding author: Steven Cataldo, Department of Anesthesia, SUNY Downstate Medical Center, 450 Clarkson Avenue Brooklyn, NY 11203, USA, Tel: +631-748-5247; E-mail: steven.cataldo@downstate.edu

\begin{abstract}
Morbidity and mortality continues to be high during rescue intubation on critically ill patients. One of the major causes for this remains severe hypoxemia, with particular risk during the critical apneic period while attempting to place a secure airway. Current practice and standard of care provides few techniques to oxygenate and no attempts to ventilate during this period of laryngoscopy and orotracheal intubation. The Nasal Oxygenation and Ventilation of the Airway (NOVA) Technique demonstrates uninterrupted nasal positive pressure ventilation throughout the intubation process in order to eliminate the apneic period and improve patient outcomes during these high-risk, critical procedures.
\end{abstract}

Keywords: NOVA; Intubation; Apneic period; Airway management; Non-invasive positive pressure ventilation; Acute respiratory failure

\section{Introduction}

Airway management in critically ill patients remains a very challenging situation. Many of these patients require NonInvasive Positive Pressure Ventilation (NIPPV) in order to maintain oxygen saturation above $92 \%$, while others are in acute respiratory failure despite NIPPV [1]. NIPPV has been shown to improve outcomes in selected patients with acute respiratory failure and Barjaktarevic and Berlin demonstrated that nasal NIPPV during awake fiberoptic intubation can maintain oxygen saturation and prevent complications associated with desaturation [2-4]. Despite this evidence, nasal NIPPV during direct laryngoscopy and intubation has never been documented in the literature.

Non-invasive positive pressure ventilation can decrease the work of breathing, improve gas exchange, recruit atelectatic lung regions, stent open obstructed airways, and match intrinsic Positive End Expiratory Pressure (PEEP). In addition, NIPPV may prevent the complications of invasive mechanical ventilation [5]. It is an established therapy for respiratory failure due to acute exacerbations of chronic obstructive pulmonary disease and cardiogenic pulmonary edema [5-7]. Early studies found a trend toward better outcomes with NIPPV [6], however, the largest study of NIPPV in hypoxemic respiratory failure was unable to confirm this benefit. In part, this is because of an increased mortality risk among patients who require rescue endotracheal intubation after NIPPV failure $[7,8]$.

The current standard of care for airway management and endotracheal intubation in emergency situations has an astonishing life-threatening complication rate of $30 \%$, along with a risk of immediate mortality at $3-4 \%$ and severe hypoxemia in $18 \%[1,9,10]$. Many patients in acute respiratory failure require NIPPV, which provides high concentrations of oxygen, inspiratory flow rates, and PEEP $[11,12]$. Therefore, patients with inadequate gas exchange despite NIPPV are at high risk of deterioration when clinicians remove NIPPV for rescue intubation. Even at a center with extensive experience, 3 of the 21 patients with acute respiratory failure had cardiac arrest during attempts to perform rescue endotracheal intubation for NIPPV failure [7].

Theoretically, maintaining positive pressure throughout the intubation process might succeed in improving patient safety. We report the Nasal Oxygenation and Ventilation of the Airway (NOVA) technique for performing laryngoscopy and orotracheal intubation while the patient undergoes uninterrupted nasal NIPPV via a resuscitator bag or ventilator with a nasal anesthesia mask. We present three cases on different patients supporting this technique and its application for those in acute respiratory failure and/or with anticipated difficult intubation. To our knowledge, this technique has not been reported elsewhere (Figure 1).

\section{Case 1}

This is a case of a 70-year-old morbidly obese woman, postop day 3 status post total abdominal hysterectomy for uterine cancer, with a recently documented Cormack-Lehane Grade II/III airway. Her history was significant for mild systolic CHF with tricuspid regurgitation who had arrived to the ICU post-op hypotensive at $80 / 40$ s, tachycardic to 140 s, with bandemia and acute renal failure. She was maintained on CPAP in the ICU; 2D 


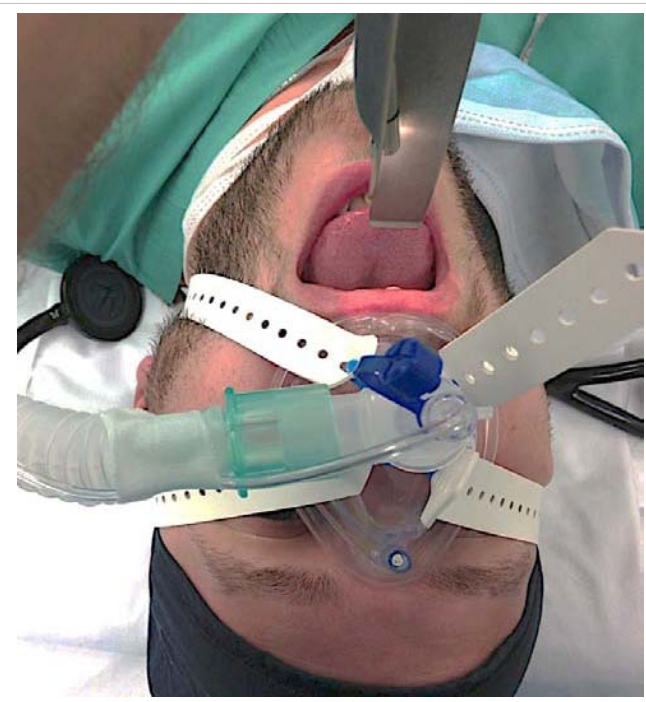

Figure 1: Representation of NOVA Technique on Volunteer Subject.

echo showed severe RV strain and pulmonary artery catheter revealed very low pulmonary wedge pressure with elevated pulmonary artery pressure consistent with pulmonary embolus. The ICU called the SUNY Downstate Anesthesia code team for an emergency intubation on post-op day 3 as she entered acute respiratory failure despite being on full facemask CPAP with $100 \% \mathrm{FiO}_{2}$. They stated that they briefly took her off CPAP to place a nasogastric tube, however they aborted because she immediately desaturated to the 70's and had rapid shallow breathing with altered mental status.

Upon arrival the patient had been placed back on full facemask CPAP with $100 \% \mathrm{FiO}_{2}$, was saturating $86 \%$ with a heart rate of 110 , had altered mental status, and was normotensive on epinephrine drip at 120/80s and heparin drip for PE. Given the patient's severe lack of physiologic respiratory reserve and history of recent significant desaturation when her full facemask CPAP was removed, the plan was to maintain positive pressure ventilation throughout the intubation process.

The code team employed a novel technique, the Nasal Oxygenation and Ventilation of the Airway (NOVA) technique, where the patient was to have uninterrupted oxygenation and ventilation throughout the intubation process. The NOVA technique utilized an anesthesia facemask placed over the patient's nose to allow access to the mouth. The resuscitation bag was connected to the left side of the mask to prevent it from obstructing the provider's view of the airway when laryngoscopy was performed. The theory was to eliminate the critical "apneic period" by utilizing NIPPV throughout the intubation attempt.

CPAP and the full facemask were discontinued and the nasal mask along with the resuscitation bag placed on the patient. During this brief time period, the patient desaturated from $86 \%$ to $75 \%$ and was subsequently assisted with bag-mask via the nose to $98 \%$. The code team continued to assist the patient during induction with $60 \mathrm{mg}$ of Lidocaine and $8 \mathrm{mg}$ of Etomidate. Adequate ventilation was established, paralysis with
$50 \mathrm{mg}$ of Rocuronium was achieved, and nasal mask NIPPV was maintained to allow for the muscle relaxant to take effect. During direct laryngoscopy, the anesthesia provider was initially unable to see the vocal cords. However, she denied that the nasal mask obstructed her view. The provider removed the laryngoscope and re-positioned the patient while another member of the team continued nasal mask NIPPV. Oxygen saturation had not dropped below $95 \%$. On second attempt the provider stated that she had a clear view of the vocal cords and successfully passed the Endotracheal Tube (ETT). An EZ cap was placed on the ETT and the resuscitation bag was switched from the nasal mask to the ETT. The patient's oxygen saturation was still 95\%, and the patient was given six breaths, which resulted in color change on the EZ cap, confirming successful ETT placement. The patient was placed on the ventilator and management continued by the ICU team.

\section{Case 2}

A 35 year old man with history of morbid obesity (BMI > 40), obstructive sleep apnea (OSA), and hypertension presented to the Emergency Department with complaints of right leg pain after falling off of a stage one week prior. On admission he was hypotensive at 95/60, tachycardic at $120 \mathrm{bpm}$ with oxygen saturation $85 \%$ despite nasal cannula $6 \mathrm{~L} \mathrm{O}_{2}$. On exam patient had no palpable popliteal pulse in the right lower extremity and Doppler ultrasound was absent of blood flow distal to the knee. CT scan revealed transected popliteal artery on the right with large hematoma. Laboratory studies were significant for severe anemia and rhabdomyolysis. At this time the anesthesia team was called for emergent intubation and an above-the-knee amputation in the operating room.

Given the patient's critical condition, acute respiratory failure despite supplemental oxygen therapy, and history of OSA coupled with morbid obesity, it was assumed that the intubation may prove difficult and that the apneic period, no matter how brief, would pose a great risk for peri-intubation complications, particularly life-threatening hypoxemia. The NOVA technique was initiated in the operating room by placing a nasal mask over his nose for pre-oxygenation using pressure support ventilation to supplement spontaneous respiration while standard ASA monitors were placed. Induction was performed with $20 \mathrm{mg}$ Etomidate along with $100 \mathrm{mg}$ Succinylcholine while maintaining nasal NIPPV by placing the anesthesia machine to pressure controlled ventilation at $20 \mathrm{~cm} \mathrm{H}_{2} \mathrm{O}$, PEEP of $10 \mathrm{~cm} \mathrm{H}_{2} \mathrm{O}$, and a rate of 14 per minute. The resident anesthesiologist attempted direct laryngoscopy but failed due to inability to visualize the vocal cords. The patient's head and neck were repositioned and successful orotracheal intubation was performed on second attempt. Nasal ventilation was continued successfully throughout the entirety of the intubation process and there were no desaturations or associated complications.

To note, the same patient returned to the operating room three days later for a wound washout under general anesthesia. The anesthesiologist was untrained in the NOVA technique and induced the patient using standard of care including preoxygenation and rapid sequence induction. The patient 
immediately desaturated during the apneic period, became severely bradycardic and required resuscitation with eventual return of effective spontaneous circulation.

\section{Case 3}

This is a case of a 43 year old man with history of hypertension, diabetes, end-stage renal disease on hemodialysis and acute stroke with altered mental status. The patient was in the ICU step-down unit when he acutely entered hypercapnic respiratory failure. The anesthesia team was called for an emergency rescue intubation. Upon arrival the patient was saturating $97 \%$ breathing spontaneously on $100 \%$ non-rebreather and his other vital signs were stable within normal limits. On exam, the patient's airway was non-reassuring secondary to short thyromental distance. Due to the anticipated difficult intubation but reassuring maskventilation, the plan was made to use the NOVA technique so as to avoid the apneic period in preparation for an extended intubation process.

As predicted, the patient proved to be a difficult intubation because of his short thyromental distance. Nasal ventilation was successfully continued throughout four intubation attempts, lasting several minutes, requiring multiple providers and including both direct and video laryngoscopy. The patient's vital signs remained stable throughout and despite the extended intubation process; there were no interruptions for bag-mask ventilation and no associated desaturations or intubation complications.

\section{Discussion}

The "apneic period," henceforth defined as the period between last spontaneous or delivered breath and successful placement of an endotracheal tube, represents one of the most critical time periods in airway management. Failure to successfully ventilate or intubate contributes to patient mortality and morbidity, including but not limited to: hypoxemia, aspiration, bradycardia, ischemia, and cardiac arrest. Therefore, it is incumbent on airway management specialists to create an environment tailored to each individual patient to ensure success. Critically ill patients represent a particularly at-risk population given their lack of pulmonary reserve and subsequently short safe apneic periods. In addition, they are more likely to have anatomical abnormalities and are performed in more uncontrolled settings with subsequent higher likelihood of difficult intubation. In general, this population has a severely reduced Functional Residual Capacity (FRC), coupled with often-increased basal metabolic rates and oxygen consumption. Current practice to prolong the safe apneic period includes pre-oxygenation and de-nitrogenation with or without positive pressure, using a ramped position in vulnerable patients, and occasional use of apneic oxygenation using high flow nasal cannula or other devices. These techniques have been widely supported in the literature and are considered routine practice for at-risk patients [13-15].

Recent literature, as previously stated, helps to support the use of continued nasal NIPPV during fiberoptic intubation in critically ill patients [2]. This study was performed on awake patients spontaneously breathing and demonstrated great triumph in avoiding critical airway management complications in this high-risk population. In their discussion, they reported that direct laryngoscopy did not seem feasible during NIPPV because the circuit, which connects to the mask, would obstruct the provider's view of the airway. To date, there exists no literature demonstrating the use of nasal NIPPV during laryngoscopy and intubation in anesthetized or paralyzed patients. Jun Oto and colleagues at Massachusetts General Hospital published a randomized controlled trial in Critical Care validating that nasal CPAP and NIPPV was more effective than full facemask in unconscious patients under general anesthesia [16]. For these reasons, the SUNY Downstate Anesthesia team felt confident that this approach could incur the highest success while reducingcomplications, and that the risks posed from use of nasal NIPPV were minimal in comparison to potential benefit. There was strong clinical evidence that the safe apneic period for the firsttwo patients described was extremely short, that NIPPV alone was proving inadequate, and that they were at exceedingly high risk for morbidity and mortality during the rescue intubation process. The third case is a classic example of reassuring mask ventilation but anticipated difficult intubation, which in turn required numerous attempts and an apneic period extending beyond the safe margin for even the healthiest of patients. The overwhelming success achieved during these rescue intubations, especially given the multiple intubation attempts and prolonged time of the intubation process, may be a milestone by which the practice of emergency and high-risk airway management changes moving forward.

The NOVA technique described here was performed on critically ill patients for rescue intubation, but its application may prove to be widespread throughout all of airway management in multiple settings. There are many mechanisms by which airway specialists can predict both the difficulty of airway management and estimation of safe apneic period. What most will agree upon is that despite proper evaluation, the risk of difficult ventilation or intubation and underestimation of safe apneic period still exists and poses a great threat to airway success and patient safety. It has been supported that nasal NIPPV may be more effective than full facemask ventilation and it has been shown that nasal NIPPV can be continued with success and with fewer complications during airway management procedures. The NOVA technique may therefore be applicable to an even larger population of at-risk patients within a variety of clinical settings, including the ICU, emergency department, non-hospital setting, and operating room. Given that the patient can be successfully ventilated, the technique described here may eliminate the apneic period altogether, therefore eliminating one of the most historically critical periods of all of airway management and improving patient outcomes.

\section{References}

1. Antonelli M, Conti G, Moro ML, Esquinas A, Gonzalez-Diaz G, Confalonier $\mathrm{M}$, et al. Predictors of failure of noninvasive positive pressure ventilation in patients with acute hypoxemic respiratory failure: a multi-center study. Intensive Care Med. 2001; 27(11): 17181728. 
2. Barjaktarevic I, Berlin D. Bronchoscopic Intubation During Continuous Nasal Positive Pressure Ventilation in the Treatment of Hypoxemic Respiratory Failure. J Intensive Care Med. 2013; Nov 15: 1-7

3. Baillard C1, Fosse JP, Sebbane M, Chanques G, Vincent F, Courouble $\mathrm{P}$, et al. Noninvasive Ventilation Improves Preoxygenation before Intubation of Hypoxic Patients, American Journal of Respiratory and Critical Care Medicine. 2006; 174(2): 171-177.

4. Nava S, Hill N. Non-invasive ventilation in acute respiratory failure. Lancet. 2009; 374(9685): 250-259. doi:10.1016/S01406736(09)60496-7.

5. Keenan SP, Sinuff T, Cook DJ, Hill NS. Ann Intern Med. 2003; 138(11): 861-870.

6. Gray A, Goodacre S, Newby DE, Masson M, Sampson F, Nicholl J. Noninvasive ventilation in acute cardiogenic pulmonary edema. $\mathrm{N}$ Engl J Med. 2008; 359(2): 142-151.

7. Delclaux C, L'Her E, Alberti C, Mancebo J, Abrouq F, Conti G, et al. Treatment of acute hypoxemic non hypercapnic respiratory insufficiency with continuous positive airway pressure delivered by a facemask-a randomized controlled trial. JAMA. 2000; 284(18): 2352 2360 .

8. Demoule A, Girou E, Richard IC, Taille S, Brochard L. Benefits and risks of success or failure of noninvasive ventilation. Intensive Care Med. 2006; 32(11): 1756-1765.

9. Jaber S, Amraoui J, Lefrant JY, Arich C, Cohendy R, Landreau L, et al. Clinical practice and risk factors for immediate complications of endotracheal intubation in the intensive care unit: A prospective, multiple-center study. Crit Care Med. 2006; 34(9): 2355-2361.

10. Thomas M. The incidence and risk factors for cardiac arrest during emergency tracheal intubation: A justification for incorporating the ASA Guidelines in the remote location. Presented in part at the Annual Meeting of the American Society of Anesthesiologists, Dallas, TX, October 9-13, 1999; and the Annual Meeting of the Society of Critical Care Medicine, February 12-15, 2000.

11. Antonelli M, Conti G, Rocco M, Bufi M, De Blasi RA, Vivino G, et al. A comparison of noninvasive positive-pressure ventilation and conventional mechanical ventilation in patients with acute respiratory failure. N Engl J Med. 1998; 339(7): 429-435.

12. Griesdale DE, Laine Bosma T, Kurth T, Isac G, Chittock DR. Complications of endotracheal intubation in the critically ill. Intensive Care Medicine. 2008; 34(10): 1835-1842. doi: 10.1007/s00134-0081205-6.

13. Weingart SD, Levitan RM. Preoxygenation and prevention of desaturation during emergency airway management. Ann Emerg Med. 2012; 59(3): 165-175. doi: 10.1016/j.annemergmed.2011.10.002.

14. Ramachandran SK, Cosnowski A, Shanks A, Turner CR. Apneic oxygenation during prolonged laryngoscopy in obese patients: a randomized, controlled trial of nasal oxygen administration. J ClinAnesth. 2010; 22(3): 164-168. doi: 10.1016/j. jclinane.2009.05.006.

15. Mort TC, Waberski BH, Clive J. Extending the preoxygenation period from 4 to 8 mins in critically ill patients undergoing emergency intubation. Crit Care Med. 2009; 37(1): 68-71. doi: 10.1097/ CCM.0b013e318192845e.

16. Jun Oto, Qian Li, William Kimball, Jingping Wang, Sabouri AS, Harrell PG. Continuous Positive Airway Pressure and Ventilation are More Effective with a Nasal Mask Than a Full Facemask in Unconscious Subjects: A Randomized Controlled Trial. Critical Care. 2013; 17(16): R300. doi: 10.1186/cc13169. 